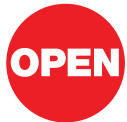

SUBJECT AREAS:

PHYSIOLOGY

AGEING

BIOENERGETICS

SKELETAL MUSCLE

Received

16 November 2012

Accepted

4 February 2013

Published

19 February 2013

Correspondence and requests for materials should be addressed to G.A.C. Igiovanni. cavagna@unimi.it)

\title{
Running humans attain optimal elastic bounce in their teens
}

\author{
Mario A. Legramandi ${ }^{1}$, Bénédicte Schepens ${ }^{2} \&$ Giovanni A. Cavagna ${ }^{1}$

\begin{abstract}
'Section of Human Physiology, Department of Pathophysiology and Transplantation (DePT), University of Milan, 20133 Milan, Italy, ${ }^{2}$ Institute of Neuroscience, Laboratoire de physiologie et biomécanique de la locomotion, Université catholique de Louvain, 1348 Louvain-la-Neuve, Belgium.
\end{abstract}

In an ideal elastic bounce of the body, the time during which mechanical energy is released during the push equals the time during which mechanical energy is absorbed during the brake, and the maximal upward velocity attained by the center of mass equals the maximal downward velocity. Deviations from this ideal model, prolonged push duration and lower upward velocity, have found to be greater in older than in younger adult humans. However it is not known how similarity to the elastic bounce changes during growth and whether an optimal elastic bounce is attained at some age. Here we show that similarity with the elastic bounce is minimal at 2 years and increases with age attaining a maximum at 13-16 years, concomitant with a mirror sixfold decrease of the impact deceleration peak following collision of the foot with the ground. These trends slowly reverse during the course of the lifespan.

ndirect evidence that energy is partly conserved in human running thanks to an elastic bounce of the body is provided by an efficiency of positive work production twice that attained by a contracting muscle ${ }^{1,2}$. The elasticity of the bounce can also be directly deduced by considering the mechanical energy changes of the center of mass of the body after landing and before takeoff $f^{3,4}$. In bouncing gaits such as running, hopping and trotting mechanical energy is absorbed each step by muscle-tendon units when the body decelerates during the brake and restored when the body reaccelerates during the push. In this stretch-shorten cycle of muscle-tendon units, some energy is stored elastically during stretching and recovered during shortening. In adult running elastic energy storage and recovery is greater the greater the length change of tendon relative to that of muscle. This is because adult tendon has a very small elastic hysteresis ${ }^{5,6}$, i.e. the force exerted during shortening is only slightly less than during stretching. Muscle's stretch-shorten cycle on the contrary exhibits a large hysteresis, i.e. a large energy loss, because it exerts a force during stretching $F_{\text {str }}$, which may largely exceed that during shortening $F_{\text {sho. }}$. The relative role of muscle vs tendon involvement during the bounce can be deduced considering that during running on the level at a constant speed the momentum lost during the brake $F_{\text {str }} t_{\text {brake }}$ equals the momentum gained during the push $F_{\text {sho }} t_{\text {push }}$, and since $F_{\text {str }}>F_{\text {sho }}$ in contracting muscle then $t_{\text {brake }}<t_{\text {push }}$ when muscle instead of tendon lengthens and shortens. The ratio between time intervals during which negative and positive work are done $t_{\text {brake }} / t_{\text {push }}$ would approach unity in an ideally elastic bounce sustained uniquely by tendon (where $\left.F_{\text {str }} \sim F_{\text {sho }}\right)$. The asymmetric response of muscle to lengthening and shortening also explains the difference between maximal downward velocity $V_{\mathrm{v}, \mathrm{mx} \text {,down }}$ (higher) and upward velocity $V_{\mathrm{v}, \mathrm{mx} \text {,up }}$ (lower) attained by the center of mass during the bounce. In fact, a higher $V_{\mathrm{v}, \mathrm{mx} \text {,down }}$ can be passively attained during the fall thanks to gravity relying, for the downward deceleration, on the greater force the muscle is able to afford when lengthening during the subsequent negative work phase (brake). On the contrary, a lower maximal upward velocity $V_{\mathrm{v}, \mathrm{mx} \text {,up }}$ is actively attained against gravity by muscular contraction during the positive work phase (push) when the muscle is shortening and is capable of a lower force. This explains why, when muscle operates instead of tendon, $V_{\mathrm{v}, \mathrm{mx}, \mathrm{up}}$ $<V_{\mathrm{v}, \mathrm{mx} \text {,down }}$. Therefore, $t_{\mathrm{brake}} / t_{\mathrm{push}}$ and $V_{\mathrm{v}, \mathrm{mx} \text {,up }} / V_{\mathrm{v}, \mathrm{mx} \text {,down }}$ are greater the more the kinetics and the kinematics of the bounce approach those of an ideal elastic body. Both ratios increase with the active muscle contraction that prevents muscle lengthening and thus favors tendon lengthening. In fact: i) $t_{\text {brake }} / t_{\text {push }}$ is lower at low running speed, when muscle activation is lower, whereas $t_{\mathrm{brake}} \sim t_{\text {push }}$ at high speeds, when muscle activation is higher ${ }^{3}$; ii) $t_{\text {brake }} / t_{\text {push }}$ is lower in old humans than in young adult humans associated with a lower force attained during the bounce by the elderly ${ }^{4,7}$.

It is not known how the running bounce differs from that of an ideal elastic body during growth. In this study we measured $t_{\text {brake, }} t_{\text {push }}, V_{\mathrm{v}, \mathrm{mx} \text {,up }}, V_{\mathrm{v}, \mathrm{mx} \text {,down }}$ and the vertical acceleration of the center of mass of the body $a_{\mathrm{v}}$ during running steps in nine age groups with mean ages ranging between 2.6 and 27.7 years. 


\section{Results}

Indicative records obtained on two subjects 2.5 years and 15.8 years old, where the difference in results between ages is largest, are shown in Fig. 1. In the younger subject:

1) $t_{\text {brake }} / t_{\text {push }}$ is lower mainly due to a shorter duration of the brake consequent to a sharper decrease of the total mechanical energy of the center of mass $E_{\text {tot }}$ following the aerial phase $t_{\mathrm{a}}$.

2) The peak in kinetic energy of vertical motion $E_{\mathrm{kv}}=0.5 M_{\mathrm{b}} V_{\mathrm{v}}{ }^{2}$ (where $M_{\mathrm{b}}$ is the mass of the body and $V_{\mathrm{v}}$ is the vertical velocity of the center of mass of the body) is lower during the lift than during the fall, indicating a lower ratio $V_{\mathrm{v}, \mathrm{mx}, \mathrm{up}} / V_{\mathrm{v}, \mathrm{mx}, \mathrm{down}}$.

3) The impact deceleration peak following landing $a_{\mathrm{v} \text {,impact }}$ is much greater than in the older subject, whereas the subsequent 'active' peak, roughly simultaneous with the minimum of $E_{\text {tot }}$ and $E_{\mathrm{p}}$, is similar to that of the older subject ${ }^{8}$.

Average values of $t_{\text {brake }} / t_{\text {push }}, V_{\mathrm{v}, \text { mx, up }} / V_{\mathrm{v}, \mathrm{mx} \text {,down }}$ and $a_{\mathrm{v}, \text { impact }}$ measured in the nine age groups of the present study are given in Table 1 and plotted as a function of age in Fig. 2. The three last rows in Table 1 (indicated by asterisks) and the open symbols in Fig. 2 refer to $t_{\text {brake }} / t_{\text {push }}$ and $V_{\mathrm{v}, \mathrm{mx}, \mathrm{up}} / V_{\mathrm{v}, \mathrm{mx} \text {,down }}$ data obtained in two previous studies $^{3,4}$ in the same speed range. It can be seen that during growth both $t_{\text {brake }} / t_{\text {push }}$ and $V_{\mathrm{v}, \mathrm{mx}, \mathrm{up}} / V_{\mathrm{v}, \mathrm{mx} \text {,down }}$ increase to a maximum at

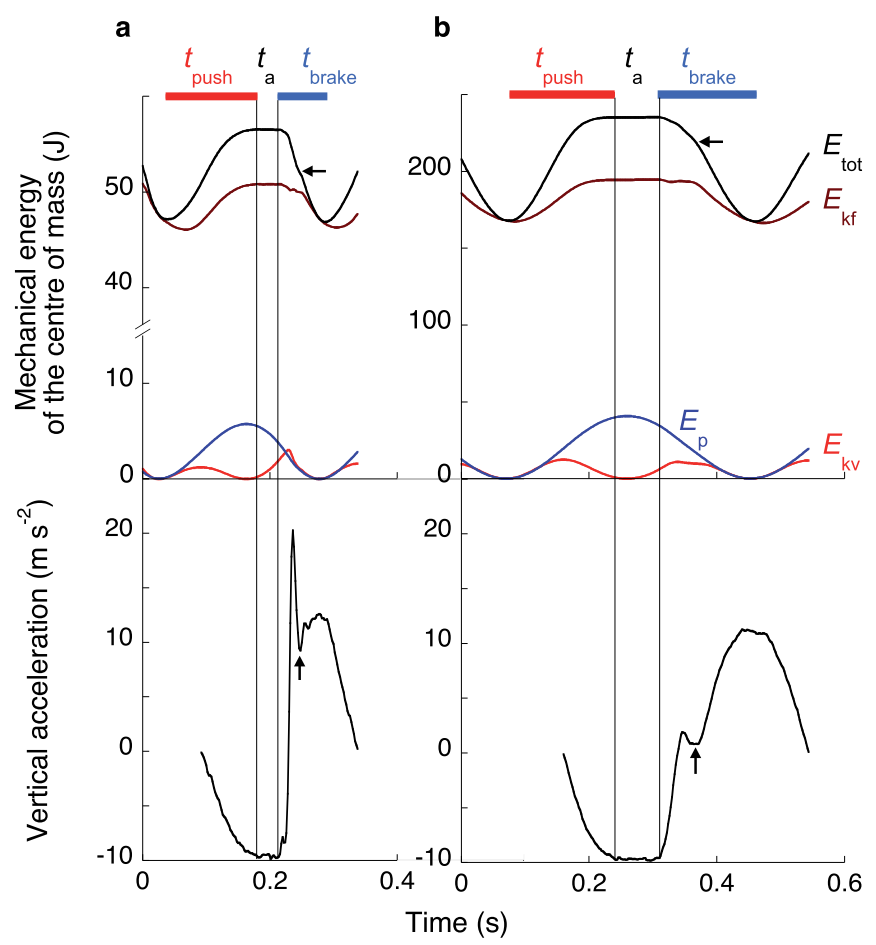

Figure $1 \mid$ Mechanical energy and vertical acceleration of the center of mass of the body in one running step of two subjects with lowest and highest similarity to an elastic bounce. (a), 2.5 years, $16.8 \mathrm{~kg}, 8.8 \mathrm{~km} \mathrm{~h}^{-1}$; (b), 15.8 years, $50.7 \mathrm{~kg}, 9.4 \mathrm{~km} \mathrm{~h}^{-1}$. $E_{\mathrm{p}}$ is the gravitational potential energy, $E_{\mathrm{kv}}$ and $E_{\mathrm{kf}}$ are the kinetic energies of vertical and forward motion,

respectively, and $E_{\mathrm{tot}}=E_{\mathrm{p}}+E_{\mathrm{kv}}+E_{\mathrm{kf}}$ is the total mechanical energy of the center of mass in a sagittal plane. Horizontal bars indicate push duration ( $t_{\text {push }}$, time interval during which $E_{\text {tot }}$ increases, red) and brake duration ( $t_{\text {brake }}$, time interval during which $E_{\text {tot }}$ decreases, blue) separated by the aerial phase $t_{\mathrm{a}}$. After the aerial phase, $E_{\text {tot }}$ decreases sharply in the younger subject resulting in a relatively shorter $t_{\text {brake }}$ and higher peak of $E_{\mathrm{kv}}$ with a greater vertical deceleration following impact of the foot on the ground. Arrows show that the fraction of $E_{\text {tot }}$ lost during the impact peak, and not available to be stored as elastic energy before the beginning of the push, is relatively greater in the younger subject.
13-16 years whereas $a_{\mathrm{v} \text {,impact }}$ decreases to a minimum at about the same age. The maximal deceleration downward $a_{\mathrm{v} \text {,impact }}$ following collision of the foot with the ground is, on average, $\sim 6$ times greater in the 2 years group than in the 16 years group (Table 1). Subsequently the ratios $t_{\text {brake }} / t_{\text {push }}$ and $V_{\mathrm{v}, \mathrm{mx} \text {,up }} / V_{\mathrm{v}, \mathrm{mx} \text {,down }}$, which would attain unity in an elastic bounce, decrease and $a_{\mathrm{v} \text {,impact }}$ increases.

\section{Discussion}

The mirroring opposite trend of the $a_{\mathrm{v} \text {,impact }}$ curve with the $t_{\mathrm{brake}} /$ $t_{\mathrm{push}}$ and $V_{\mathrm{v}, \mathrm{mx} \text {,up }} / V_{\mathrm{v}, \mathrm{mx} \text {, down }}$ curves in Fig. 2 strongly suggests that the impact peak is a relevant factor impeding an elastic bounce. This is reasonable because some of the mechanical energy absorbed and released by the heel pad and other structures during the impact phase $^{9}$ is lost prior the beginning of the push, thus decreasing the mechanical energy at disposal for the subsequent positive work phase. The fall in $E_{\text {tot }}$ during $t_{\text {brake }}$ represents the total amount of energy that can possibly be stored elastically. In the example of Fig. 1, the impact duration occupies $\sim 45 \%$ of the total fall in $E_{\text {tot }}$ in the 2.5 years old subject and $\sim 24 \%$ in the 15.8 years old subject. It follows that relatively less mechanical energy is left after the impact phase to be stored in muscle-tendon units of the younger subject during the fall in $E_{\text {tot. }}$. The mechanical energy lost during the impact phase must be replaced by muscular contraction during the following positive work phase resulting, as described above, in an increased duration of $t_{\text {push }}$ and in a decrease of $V_{\mathrm{v}, \mathrm{mx} \text {,up }}$, i.e. in a less elastic bounce and a greater energy expenditure. In fact, measurements made in a previous study ${ }^{2}$ show that the efficiency of positive work production during running below $11 \mathrm{~km} \mathrm{~h}^{-1}$ is lower in 4.5 years old children than in 21.6 years adults $(0.405 \pm 0.046$ (s.d.), $N=46$ vs. $0.426 \pm 0.036$ (s.d.), $N=67, P=0.014$ ).

This study draws attention to two points: $i)$ the youngest subjects are more exposed to high-impact collisions, and ii) the impact peak and the similarity to an elastic bounce change during growth.

The first point has practical health implications. It is known that high-impact collision forces are likely to be associated with injuries of the muscular-skeleton system ${ }^{10-12}$. The present finding, that the impact peak during running is elevated in the youngest subjects requires particular attention.

With regard to the second point it is relevant to consider that the mass-specific vertical stiffness of the running bounce $k / M_{\mathrm{b}}$ decreases during growth to a minimum in the 16 years group to increase again in the 28 years group ${ }^{8}$ with the same trend of the impact peak found in the present study. In other words, the step frequency is higher in the youngest, due to the lower dimensions of their body, requires a higher mass-specific vertical stiffness, to cope with the natural frequency of the bouncing system ${ }^{8}$, and this, in turn, causes a higher impact peak and a lower similarity to an elastic bounce. Interestingly, an inverse relationship between $k / M_{\mathrm{b}}$ and similarity to an elastic bounce was also found when comparing running, trotting and hopping animals of different size ${ }^{13}$. In adult humans, the height of the impact peak increases with the effective foot mass $M_{\text {eff }}$, i.e. with the proportion of the body mass $M_{\mathrm{b}}$ stopping abruptly at touch-down ${ }^{9,14}$. We do not know studies describing changes of the ratio $M_{\text {eff }} / M_{\mathrm{b}}$ during growth: this requires further experiments. However, it is worth noting since now that the optimal similarity to an elastic bounce is attained in the 16 years group thanks to an increase in $M_{\mathrm{b}}$ not compensated by an increase in $k$ (ref. 8), resulting in a more compliant system, and it is abandoned in the 28 years group because of an increase in $k$ not compensated by the increase in $M_{\mathrm{b}}$, resulting in a stiffer system. This suggests that the completion of neuro-muscular maturation ${ }^{15}$ associated with greater tendon compliance ${ }^{16}$ attains in the teens an ideal condition that is no longer tenable at an older age.

It remains to be explained why a stiffer system results in a less elastic bounce and if this condition is necessarily caused, in animals 


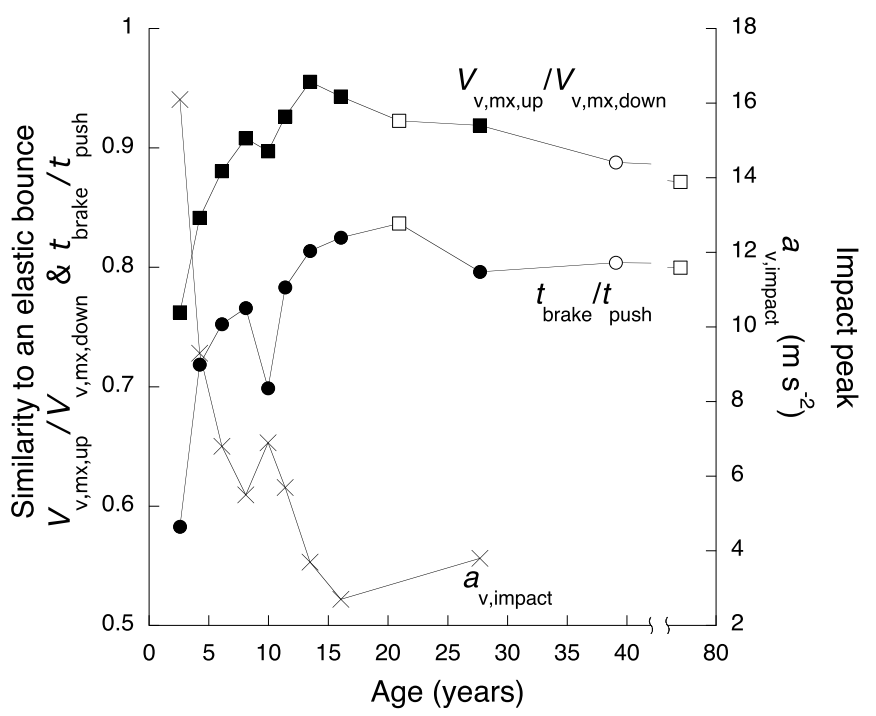

Figure $2 \mid$ Effect of age on the similarity to an elastic bounce and on the deceleration peak following impact of the foot on the ground. The similarity to an elastic bounce, which is greater the higher the ratios $t_{\text {brake }} /$ $t_{\text {push }}$ and $V_{\mathrm{v}, \mathrm{mx} \text {,up }} / V_{\mathrm{v}, \mathrm{mx} \text {,down }}$, increases during growth, attains a maximum in the teens and subsequently decreases. This trend is mirrored by an opposite trend of the impact peak following collision of the foot on the ground after the aerial phase. Symbols are average values (Table 1) measured in the present study (filled squares, circles and crosses) and in two previous studies (open squares ${ }^{4}$ and circles $^{3}$ ).

as in humans, by a greater impact peak following collision with the ground. In addition, do we really know that a greater kinematic and kinetic similarity of the bounce with that of an ideal elastic body translates into a more efficient run? Not necessarily, but experimental data show that in human running: i) the efficiency is greater at high speeds ${ }^{1,2}$ when $t_{\text {brake }} \sim t_{\text {push }}$ as in an elastic bounce ${ }^{3}$; ii) the greater deviation from the elastic model in the elderly ${ }^{4}$, i.e. a lower ratio $t_{\text {brake }} / t_{\text {push }}$, is associated with a greater energy expenditure ${ }^{17}$; iii) as mentioned above, both the efficiency ${ }^{2}$ and the similarity to an elastic bounce (Fig. 2), increase from 4.5 to 21.6 years; and iv) in running turkeys and rhea, and hopping springhare and kangaroos, a lower efficiency in the smaller animal was found to be bound to a lower $t_{\text {brake }} / t_{\text {push }}{ }^{13,18}$. The lower efficiency in smaller animals was ascribed in the literature to a less efficient elastic energy storage possibly due to their tendons being relatively thicker than those of larger animals ${ }^{19-22}$.

\section{Methods}

Measurements were made starting from records of the force exerted by the foot on the ground in vertical and fore-aft directions obtained in a previous study ${ }^{8}$ by means of a force-platform. The method of analysis of the force records to obtain the mechanical energy of the center of mass of the body (Fig. 1) has been described in detail previously $y^{4,13}$. Here we used only runs where: i) the ratio between positive and negative work done during the step to maintain the motion of the center of mass was between 0.75 and 1.25 ; ii) the curves of gravitational potential energy and of the kinetic energy of forward motion were in phase with an energy transfer between them $\leq 10 \%$, warranting the mechanism of running rather than that of walking, and iii) the ratio between the average vertical force in the complete steps used for the analysis and the weight of the body was between 0.97 and 1.03. Analysis was restricted to running speeds less than $11 \mathrm{~km} \mathrm{~h}^{-1}$ because below this speed the mean vertical acceleration during the push is independent of body size and age ${ }^{8}$. Written informed consent of the subjects and/or their parents was obtained. Experiments involved no discomfort, were performed according to the Declaration of Helsinki and approved by the local ethics committee.

1. Cavagna, G. A., Saibene, F. P. \& Margaria, R. Mechanical work in running. J. Appl. Physiol. 19, 249-256 (1964).

2. Schepens, B., Willems, P. A., Cavagna, G. A. \& Heglund, N. C. Mechanical power and efficiency in running children. Pflugers Arch. 442, 107-116 (2001). 
3. Cavagna, G. A. The landing-take-off asymmetry in human running. J. Exp. Biol. 209, 4051-4060 (2006).

4. Cavagna, G. A., Legramandi, M. A. \& Peyré-Tartaruga, L. A. The landing-take-off asymmetry of human running is enhanced in old age. J. Exp. Biol. 211, 1571-1578 (2008).

5. Ker, R. F. Dynamic tensile properties of the plantaris tendon of sheep (Ovis aries). J. Exp. Biol. 93, 283-302 (1981).

6. Alexander, R. M. Tendon elasticity and muscle function. Comp. Biochem. Physiol. 133A, 1001-1011 (2002).

7. Cavagna, G. A., Legramandi, M. A. \& Peyré-Tartaruga, L. A. Old men running: mechanical work and elastic bounce. Proc. R. Soc. London Ser. B 275, 411-418 (2008).

8. Schepens, B., Willems, P. A. \& Cavagna, G. A. The mechanics of running in children. J. Physiol. (Lond) 509, 927-940 (1998).

9. Chi, K. J. \& Schmitt, D. Mechanical energy and effective foot mass during impact loading of walking and running. J. Biomech. 38, 1387-1395 (2005).

10. Gill, H. S. \& O'Connor, J. J. Heelstrike and the pathomechanics of osteoarthrosis: a pilot gait study. J. Biomech. 36, 1625-1631 (2003).

11. Milner, C. E., Ferber, R., Pollard, C. D., Hamill, J. \& Davis, I. S. Biomechanical factors associated with tibial stress fractures in female runners. Med. Sci. Sports Exerc. 38, 323-328 (2006).

12. Pohl, M. B., Hamill, J. \& Davis, I. S. Biomechanical and anatomical factors associated with a history of plantar fasciitis in female runners. Clin. J. Sports Med. 19, 372-376 (2009).

13. Cavagna, G. A. \& Legramandi, M. A. The bounce of the body in hopping, running and trotting: different machines with the same motor. Proc. R. Soc. London Ser. B 276, 4279-4285 (2009)

14. Lieberman, D. E. et al. Foot strike patterns and collision forces in habitually barefoot versus shod runners. Nature 463, 531-535 (2010).

15. Dotan, R., Cameron, M., Rotem, C., Klentrou, P., Gabriel, D. \& Falk, B. Child-adult differences in muscle activation. A Review. Pediatr. Exerc. Sci. 24, 2-21 (2012).

16. Kubo, K., Kanehisa, H., Kawakami, Y. \& Fukanaga, T. Growth changes in the elastic properties of human tendon structures. Int. J. Sports Med. 22, 138-143 (2001).

17. Evans, S. L., Davy, K. P., Stevenson, E. T. \& Seals, D. R. Physiological determinants of $10-\mathrm{km}$ performance in highly trained female runners of different ages. J. Appl. Physiol. 78, 1931-1941 (1995).
18. Cavagna, G. A., Heglund, N. C. \& Taylor, C. R. Mechanical work in terrestrial locomotion: two basic mechanisms for minimizing energy expenditure. Am. J. Physiol. 233, R243-R261 (1977).

19. Biewener, A. A., Alexander, R. McN. \& Heglund, N. C. Elastic energy storage in the hopping of kangaroo rats (Dipodomys spectabilis). J. Zool. 195, 369-383 (1981).

20. Bennett, M. B. \& Taylor, G. C. Scaling of elastic strain energy in kangaroos and the benefits of being big. Nature 378, 56-59 (1995).

21. Alexander, R. McN., Jayes, A. S., Maloiy, G. M. O. \& Wathuta, E. M. Allometry of the leg muscles of mammals. J. Zool. 194, 539-552 (1981).

22. Pollock, C. M. \& Shadwick, R. E. Allometry of muscle, tendon, and elastic energy storage capacity in mammals. Am. J. Physiol. 266, R1022-R1031 (1994).

\section{Acknowledgements}

The authors wish to thank Andrea Cavagna for constructive discussions and suggestions on the manuscript preparation. This study was supported by the Fonds National de la Recherche Scientifique of Belgium and by the Italian Ministero dell'Istruzione, dell'Università e della Ricerca.

\section{Author contributions}

M.A.L. suggested to apply the landing-takeoff software ${ }^{3,4,13}$ to the force-platform records obtained in a previous study ${ }^{8}$, did most of the software arrangements and of the analysis of the data. B.S. provided the original force-platform records with instructions for their analysis and mechanical efficiency data, did most of the literature search and provided useful suggestions in the preparation of the manuscript. G.A.C. collaborated with software preparation, did some of the analysis of the data and wrote the paper.

\section{Additional information}

Competing financial interests: The authors declare no competing financial interests. License: This work is licensed under a Creative Commons Attribution-NonCommercial-NoDerivs 3.0 Unported License. To view a copy of this license, visit http://creativecommons.org/licenses/by-nc-nd/3.0/

How to cite this article: Legramandi, M.A., Schepens, B. \& Cavagna, G.A. Running humans attain optimal elastic bounce in their teens. Sci. Rep. 3, 1310; DOI:10.1038/srep01310 (2013) 